\title{
Investigation of urinary components in rat model of ketamine- induced bladder fibrosis based on metabolomics
}

\author{
Haozhen $\mathrm{Li}^{1}{ }^{\wedge}$, Quan $\mathrm{Zhu}^{1}$, Kaixuan $\mathrm{Li}^{1}$, Ziqiang $\mathrm{Wu}^{1}$, Zhengyan Tang ${ }^{1,2}$, Zhao Wang ${ }^{1 \wedge}$ \\ ${ }^{1}$ Department of Urology, Xiangya Hospital, Central South University, Changsha, China; ${ }^{2}$ Hunan Provincial Engineering Laboratory for Diagnosis \\ and Treatment of Genitourinary System Disease, Changsha, China \\ Contributions: (I) Conception and design: Z Wang, Z Tang, H Li; (II) Administrative support: Z Wang, Z Tang; (III) Provision of study materials \\ or patients: None; (IV) Collection and assembly of data: H Li, Q Zhu, K Li; (V) Data analysis and interpretation: H Li, Z Wang; (VI) Manuscript \\ writing: All authors; (VII) Final approval of manuscript: All authors. \\ Correspondence to: Zhengyan Tang. Department of Urology, Xiangya Hospital, Central South University, Changsha 410008, China; Hunan Provincial \\ Engineering Laboratory for Diagnosis and Treatment of Genitourinary System Disease, Changsha 410008, China. Email: xytzyan@163.com; Zhao \\ Wang. Department of Urology, Xiangya Hospital, Central South University, Changsha 410008, China. Email: xywangz07@163.com.
}

Background: Ketamine abuse has been linked to the system's damage, presenting with lower urinary tract symptoms (LUTS). While the pathogenesis of ketamine-induced urinary damage is not fully understood, fibrosis is believed to be a potential mechanism. A metabolomic investigation of the urinary metabolites in ketamine abuse was conducted to gain insights into its pathogenesis.

Methods: A rat model of ketamine induced bladder fibrosis was established through tail vein injection of ketamine hydrochloride and control group was established through tail vein injection of the equivalent normal saline. Hematoxylin and eosin $(\mathrm{H} \& \mathrm{E})$ staining and Masson trichrome staining were performed to evaluated bladder pathology. Urinary components were detected based on a metabolomic approach using ultra-high performance liquid tandem chromatography quadrupole time of flight mass spectrometry (UHPLC-QTOFMS platform). Orthogonal projections analyzed the data to latent structures discriminant analysis (OPLS-DA) and bioinformatics analysis.

Results: The rat model of ketamine induced bladder fibrosis was confirmed through H\&E and Masson trichrome staining. There were marked differences in the urinary metabolites between the experimental group and the control group. Compared to the control group, 16 kinds of differential metabolites were upregulated and 102 differential metabolites were down-regulated in the urine samples of the ketamine group. Bioinformatics analysis revealed the related metabolic pathways.

Conclusions: Using a ketamine-induced bladder fibrosis rat model, this study identified the differential urinary metabolites expressed following ketamine treatment. These results provide vital clues for exploring the pathogenesis of ketamine-induced LUTS and may further contribute to the disease's diagnosis and treatment.

Keywords: Ketamine; bladder fibrosis; metabolomics

Submitted Aug 26, 2020. Accepted for publication Dec 21, 2020.

doi: $10.21037 /$ tau-20-1202

View this article at: http://dx.doi.org/10.21037/tau-20-1202

\footnotetext{
^ ORCID: Haozhen Li, 0000-0003-0859-1960; Zhao Wang, 0000-0003-1980-2866.
} 


\section{Introduction}

Ketamine is a derivative of phencyclidine and has been used as an anesthetic in clinical practice since the 1960s (1). It was found to have hallucinogenic and psychoactive effects on ketamine abusers (2). In 2007, Shahani et al. were the first to report the lower urinary tract symptoms (LUTS) caused by ketamine abuse. The symptoms included severe dysuria, frequency, urgency, and gross hematuria (3). Severe clinical cases can present with thickening of the bladder wall and bladder contracture. In emergency cases, hydronephrosis, uremia, and other serious complications have also been reported (4-6). Life quality would be seriously affected by refractory LUTS, and the abusers may even encounter life-threatening situation (7). Some pathological studies have revealed bladder fibrosis in the ketamine abusers. Also, animals injected with ketamine can generate ketamine associated cystitis model (8-10). However, the pathogenesis of ketamine-induced LUTS is not fully understood. Furthermore, treatment regimens are limited, especially in patients with serious bladder contracture and renal insufficiencies. Therefore, it is essential to elucidate the mechanisms of ketamine-associated bladder fibrosis to improve chronic ketamine abusers' prognosis.

Pathological studies have demonstrated urothelial ulceration, erosion, tearing and infiltration of inflammatory cells in the bladder of patients with ketamine-associated LUTS (11). Several investigations have suggested that the direct or indirect effects of ketamine and its urinary metabolites may be important in the pathogenesis of bladder inflammation, fibrosis, subsequent lesions $(7,11)$. Therefore, this study examined the differential metabolites in the urine samples of rats with ketamine-induced bladder fibrosis. This will provide important insights into the pathogenesis of bladder fibrosis and facilitate its early detection by assessing the differential metabolites' levels.

Metabolomics is an important branch of systems biology to identify different metabolite expression via highthroughput methods (12). Metabolomics is divided into non-targeted metabolomics and targeted metabolomics. The former of which enables extensive screening of metabolites (13). High throughput detection of metabolomics can efficiently accomplish the qualitative and quantitative analyses of metabolites, and can further recognize the differential expression of metabolites in various groups. Identification of the differential metabolites will contribute to understanding the pathogenesis of ketamine-associated LUTS and may be applied to the disease's diagnosis.
In this study, we established a rat model of ketamine induced bladder fibrosis. After confirming the fibrosis of bladder, we detect the differential urine metabolites and associated pathways based on metabolomics.

We present the following article in accordance with the ARRIVE reporting checklist (available at http://dx.doi. org/10.21037/tau-20-1202).

\section{Methods}

\section{Rat model and the collection of urine samples}

Experiments were performed under a project license (No. 2018sydw0225) granted by experimental animal welfare center in Central South University, Hunan, China, in compliance with China's "Animal management regulations" for the care and use of animals.

Female 8-week-old Sprague-Dawley (SD) rats (200-250 g) were purchased from Hunan Slack Jingda Experimental Animal Corporation (Hunan, China). The rats were housed in a specific pathogen-free (SPF) environment at room temperature $\left(20-26^{\circ} \mathrm{C}\right)$ in $40-70 \%$ humidity under a 12-hour/12-hour light/dark cycle, and given free access to food and water.

To establish a rat model of ketamine-induced bladder fibrosis, $20 \mathrm{SD}$ rats were randomly divided into two groups, with 10 rats in each group. In the control group, $2 \mathrm{~mL}$ normal saline was injected daily into the animals' tail vein. In the experimental group, a $0.1 \mathrm{~g} / 2 \mathrm{~mL}$ ketamine hydrochloride solution (Fujian Gutian Pharmaceutical Co. Ltd.) was injected daily into each rat's tail vein. After 8 weeks of injections, urine was collected every morning for the first 2 hours for 3 consecutive days. After collection, the urine samples were transferred to frozen tubes for centrifugation $\left(4{ }^{\circ} \mathrm{C}, 10,000 \mathrm{rpm}, 10 \mathrm{~min}\right.$ ) and placed in liquid nitrogen for cryopreservation before analysis. The rats were anesthetized by pentobarbital injection $(2 \%, 40 \mathrm{mg} / \mathrm{kg})$ and sacrificed by cervical dislocation. After adequate perfusion of systemic tissues through cardiac infusion with $4 \%$ paraformaldehyde, bladder tissues were immediately removed and transferred to $4 \%$ paraformaldehyde for fixation.

\section{Histopathological observation}

Hematoxylin and eosin (HE) staining and Masson trichrome staining were performed to examine the bladder tissue histopathology. After 24 hours of fixation in $4 \%$ paraformaldehyde, bladder tissues were embedded in 
wax blocks. Following this, $4 \mu \mathrm{m}$ sections were dewaxed, rehydrated, and stained. A standard $\mathrm{HE}$ and Masson trichrome staining procedure was performed according to previous protocols $(14,15)$.

\section{Differential metabolomics detection}

For differential metabolomic analyses, $100 \mu \mathrm{L}$ of urine sample was mixed with $400 \mu \mathrm{L}$ extract solution (acetonitrile:methanol =1:1) containing an internal standard (L-2-chlorophenylalanine, $2 \mu \mathrm{g} / \mathrm{mL}$ ). The samples were sonicated for $10 \mathrm{~min}$ in ice-water bath after $60 \mathrm{~s}$ vortex. Then the samples were incubated at $-40{ }^{\circ} \mathrm{C}$ for 1 hour and centrifuged at $10,000 \mathrm{rpm}$ at $4{ }^{\circ} \mathrm{C}$ for $15 \mathrm{~min}$. The samples were mixed with $200 \mu \mathrm{L}$ of $50 \%$ acetonitrile after drying, sonicated on ice for ice and centrifuged at 13,000 rpm for $15 \mathrm{~min}$ at $4{ }^{\circ} \mathrm{C}$. Furthermore, $10 \mu \mathrm{L}$ of each sample were mixed to be the quality control (QC) sample.

The Agilent 1290 Infinity series ultra-high performance liquid chromatography (UHPLC) System (Agilent Technologies) works with UPLC BEH Amide column $(2.1 \times 100 \mathrm{~mm}, 1.7 \mu \mathrm{m})$. The mobile phase A consisted of ammonium acetate and ammonia hydroxide. The mobile phase B consisted of acetonitrile. Elution gradient conditions were as follows: $0-0.5 \mathrm{~min}, 95 \% \mathrm{~B}$; $0.5-7.0 \mathrm{~min}$, 95-65\% B; 7.0-8.0 min, 65-40\% B; 8.0-9.0 min, 40\% B; 9.0-9.1 min, 40-95\% B; 9.1-12.0 min, 95\% B. The autosampler temperature was $4{ }^{\circ} \mathrm{C}$. The column temperature was $25^{\circ} \mathrm{C}$ and the injection volume was $1 \mu \mathrm{L}$ (positive) or $1 \mu \mathrm{L}$ (negative).

\section{Data analysis}

The mass spectrometry (MS) raw data (.wiff) files were converted to the mzXML format by ProteoWizard, and processed by $\mathrm{R}$ package XCMS (version 3.2). The procedure consisted of peak deconvolution, alignment and integration. The Minfrac index was 0.5 and the cut-off index was 0.6. MS2 database in-house was applied for the identification of metabolites. The Kyoto Encyclopedia of Genes and Genomes (KEGG, www.kegg.jp) online database was used to explore the metabolic pathways and function.

\section{Statistical analysis}

The experimental data were analyzed by SPSS statistical software (version 20.0). The measurement data were expressed as mean \pm standard deviation and there are
10 biological replications in each group. Data were statistically analyzed at $\mathrm{P}$ value less than 0.05 .

\section{Results}

\section{Hematoxylin and eosin staining and Masson trichrome staining of bladder tissue}

After consecutive 8 weeks intravenous injection of ketamine, bladder specimens were analyzed through $\mathrm{HE}$ and Masson trichrome staining. Representative bladder tissue sections are presented in Figure 1. Compared to the control group (Figure $1 A$ ), the bladder specimens in the ketaminetreated group showed that the urothelium was denuded, accompanied by vascular congestion and inflammatory cell infiltration (Figure $1 B$ ). Bladder fibrosis was also confirmed through Masson trichrome staining (Figure 1D). Extracellular matrix (ECM) and collagen deposition were obvious in the ketamine treated group compared to the control group.

\section{Orthogonal projections to latent structures-discriminant analysis (OPLS-DA)}

In order to visualize and further analysis the data, the results were examined using the OPLS-DA test. Orthogonal variables that were not related to the categorical variables were filtered out. to obtain more reliable group differences for the metabolites (Figure 2). The results indicated that all of the samples in both groups were in the $95 \%$ confidence interval.

The OPLS-DA permutation test was used to evaluate the model's validity using the $\mathrm{R}^{2} \mathrm{Y}$ (model interpretability of categorical variable $\mathrm{Y}$ ) and $\mathrm{Q}^{2}$ (model predictability) values obtained after cross-validation. In addition, different random $\mathrm{Q}^{2}$ values were obtained by indiscriminately changing the order of the categorical variable $\mathrm{Y}$ several times (Figure 2). The results showed that the established model was consistent with the real sample data. Further analysis revealed that the original model demonstrated good robustness without any evidence of overfitting.

\section{Expression of differential metabolites}

The detected differential metabolites were further screened though statistical analysis. The criteria were as following: Student's $t$-test $\mathrm{P}$ value less than 0.05 , and variable importance in the projection (VIP) value of OPLS-DA model was greater 

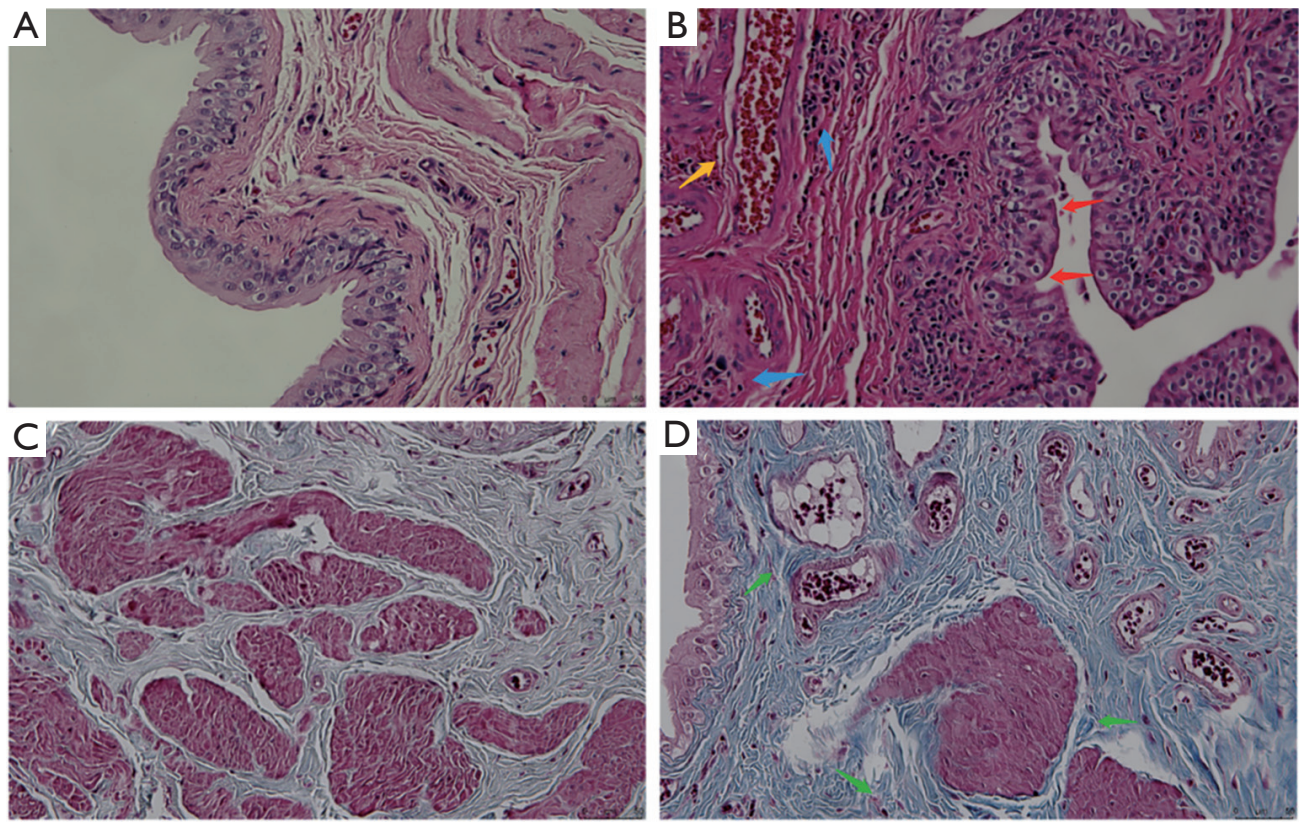

Figure 1 Histopathological changes of bladder specimen induced by 8-week ketamine injection in rat. Comparison between the control group (A) and ketamine group (B) through HE staining showed urothelium desquamation (red arrow), inflammatory cell infiltration (blue arrow), vascular distension and congestion (yellow arrow) in ketamine group. Bladder fibrosis was confirmed via Masson trichrome staining in control (C) and ketamine group (D). It showed collagen deposition (green arrow). Original magnification $\times 100$.
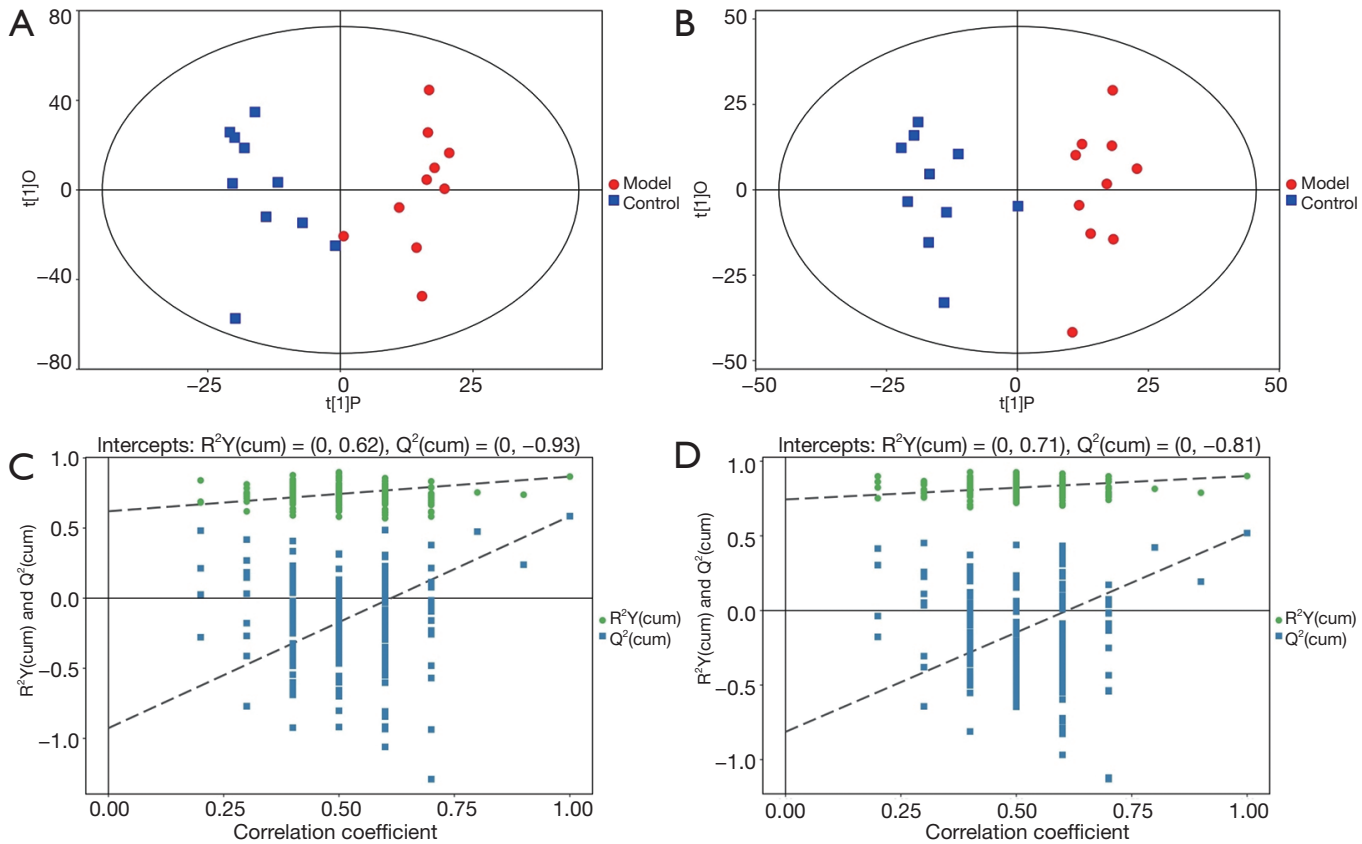

Figure 2 Score scatter plot of OPLS-DA model for ketamine group vs. control group (A for negative ion and B for positive ion mode) and permutation test of OPLS-DA model for ketamine group $v s$. control group (C for negative ion and D for positive ion mode). OPLS-DA, orthogonal projections to latent structures-discriminant analysis. 

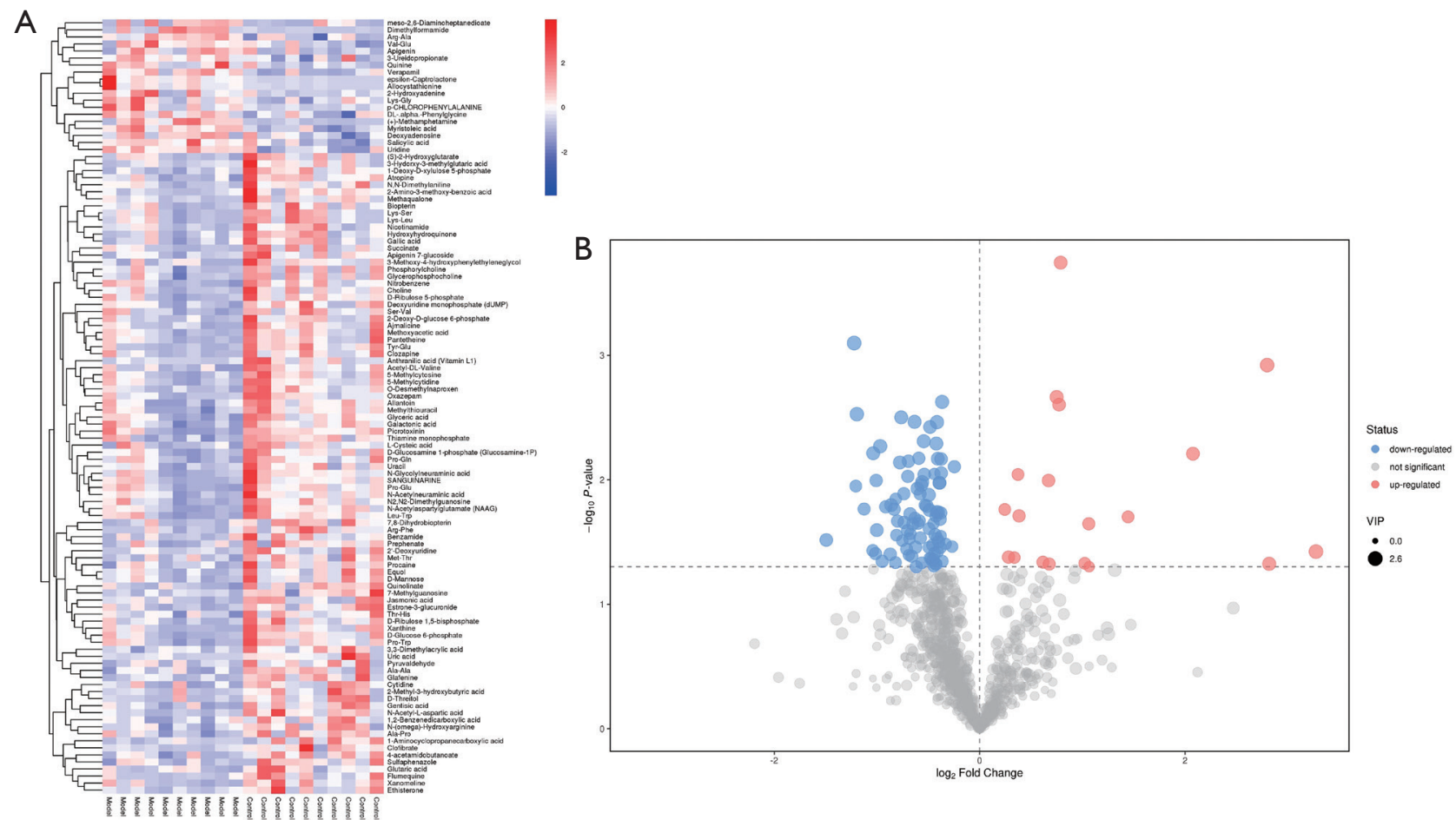

Figure 3 Comparison of all differentially expressed metabolites levels in the ketamine and control groups. Heatmaps showed 118 significantly altered metabolites between these two groups (A). The colors correspond to the abundance value of each metabolite. Volcano map showed differentially expressed metabolites screened by VIP value $>1$ and $\mathrm{P}<0.05$ (B). VIP, variable importance in the projection.

than 1 . There were 118 differential metabolites identified according to the qualitative matching analysis of the secondary MS (Table S1). Heatmaps and volcano maps were used to represent the screened differential metabolites (Figure 3). Out of the 118 differential metabolites, 16 were up-regulated and 102 were down-regulated (Table 1). The screened differential metabolites included myristoleic acid, L-homoserine, methamphetamine, dimethylformamide, anthranilic acid (vitamin L1), and others (Table 1).

Also, receiver operating characteristic (ROC) curves and box plots were used to find differential metabolites that were correlated with fibrosis trends. A total of 9 metabolites were identified with an area under the curve of ROC (AUC) greater than 0.9 (Figure 4). These differential expressed metabolites may be potential biomarkers in a rat model of ketamine induced bladder fibrosis.

\section{Bioinformatics analysis of the differential expressed metabolites}

The degree of correlation was measured by the correlation coefficient $r$, with positive correlations ranging from 0 to 1 and negative correlations ranging from 0 to -1 . When the absolute value of $\mathrm{r}$ is closer to 1 , a stronger correlation of the different metabolites is indicated. The results are presented as heatmaps (Figure $5 A$ ). To explore the relationship between metabolites and regulatory networks, metabolites were located based on KEGG database with relative metabolic responses, metabolic pathways and regulatory proteins. (Figure $5 B, C$ ). Based on the KEGG database, networks describing the metabolic and physiological processes were shown in Figure 5D,E. The enriched pathway included biosynthesis of amino acids, pyrimidine metabolism, and more (Table 2).

\section{Discussion}

In this study, a rat model of ketamine induced bladder damage and fibrosis was established by tail vein injection of ketamine. The model was confirmed by HE and Masson trichrome staining. Also, this report investigated the differential expression of urinary metabolites based on a metabolomic 
Table 1 Partial results of differential metabolites in ketamine treated rat urine samples compared to control group

\begin{tabular}{llc}
\hline Metabolites & VIP & Fold change \\
\hline Hydroxyphenyllactic acid & 2.63 & 17.03 \\
Epsilon-caprolactone & 2.48 & 9.59 \\
Allocystathionine & 2.17 & 7.02 \\
(+)-Methamphetamine & 2.35 & 6.95 \\
Dimethylformamide & 1.94 & 4.21 \\
Myristoleic acid & 2.10 & 1.73 \\
L-homoserine & 1.75 & 1.55 \\
Estrone-3-glucuronide & 2.10 & 0.49 \\
1-aminocyclopropanecarboxylic acid & 1.79 & 0.46 \\
N-acetyl-L-aspartic acid & 2.40 & 0.44 \\
Tyr-Glu & 2.12 & 0.43 \\
anthranilic acid (vitamin L1) & 1.85 & 0.36 \\
\hline
\end{tabular}

VIP, variable importance in the projection.

approach. A total of 118 differential expressed metabolites in urine samples were detected through metabolomic methods. Among them, hydroxyphenyllactic acid, epsilon-caprolactone, allocystathionine, methamphetamine, dimethylformamide, etc., were expressed differently, and the highest fold change reached to as high as 17 . Myristoleic acid has been reported to induce apoptotic necrosis of prostate cells (16), L-homoserine may be involved in the induction of regulatory $\mathrm{T}$ cells (17), and dimethylformamide has been showed to be directly toxic to the kidneys (18). Some studies have been reported that the metabolites in urine may infiltrate into bladder urothelium, related to bladder damage (19). These results suggest that ketamine-induced bladder fibrosis may be related to its direct damage or metabolites, and immunomodulation. may be a potential pathogenesis. Also, methamphetamine, one of the differential metabolites in the ketamine-treated rats, has been reported to be involved in the urinary system's impairment. These urinary metabolites should be considered when analyzing the mechanisms of ketamine-associated urinary dysfunction $(20,21)$.

There is currently no standardized method for establishing a rat model of ketamine induced urinary dysfunction, according to current literature the treatment concentrations were ranging from 5 to $60 \mathrm{mg} / \mathrm{kg}$ and treatment duration was ranging from 2 weeks to 4 months $(9,22,23)$. In this study, animal model was established through tail vein injection of ketamine at $25 \mathrm{mg} / \mathrm{kg}$ for 8 consecutive weeks.
Histopathological staining demonstrated bladder urothelial denudation, inflammatory cells infiltration, vasodilatation and extensive ECM deposition in the submucosa layer and between the detrusor muscle bundles, which indicated bladder inflammation and fibrosis. However, during 8 weeks of ketamine treatment, some rats were comatose or even died after ketamine rapid injection, which could be due to the quick increase of blood ketamine concentration through intravenous injection. In the further study, different treatment methods and duration should be explored to develop improved animal model protocol or guideline.

Based on bioinformatics analysis, differential metabolites are enriched in pathways such as pyrimidine metabolism, amino sugar and nucleotide sugar metabolism, ATP-binding cassette $(\mathrm{ABC})$ transporters, phenylalanine, tyrosine and tryptophan biosynthesis and biosynthesis of amino acids. Previous research highlighted that KRP-103 in pyrimidine metabolism is associated with bladder dysfunction (24). In distention-induced rhythmic bladder contractions (RBCs) in urethane-anesthetized guinea pigs, KRP-103 injected by intravenous dose-dependently increased the shutdown time of RBCs and decreased the amplitude of RBCs (24). Bicyclic pyrimidine derivatives may be a candidate therapeutic drug for the treatment of overactive bladder (24). Another study also indicated a correlation between phenylalanine and liver fibrosis (25). Combining metabolic pathways may provide novel ideas for studying bladder dysfunction 

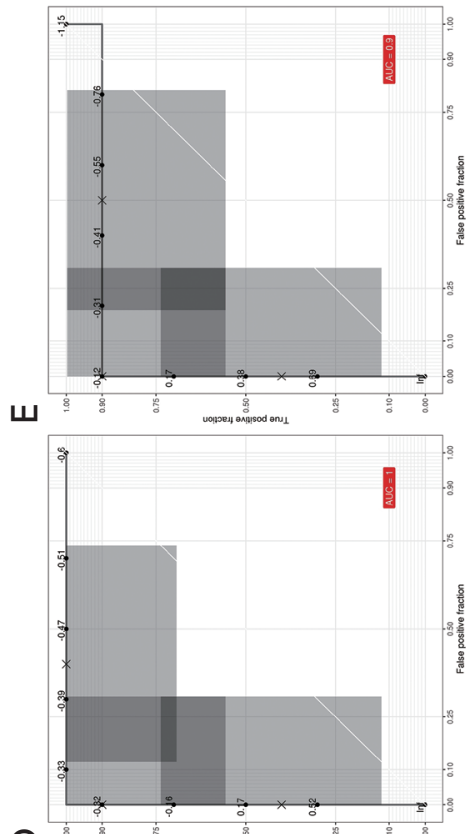

口
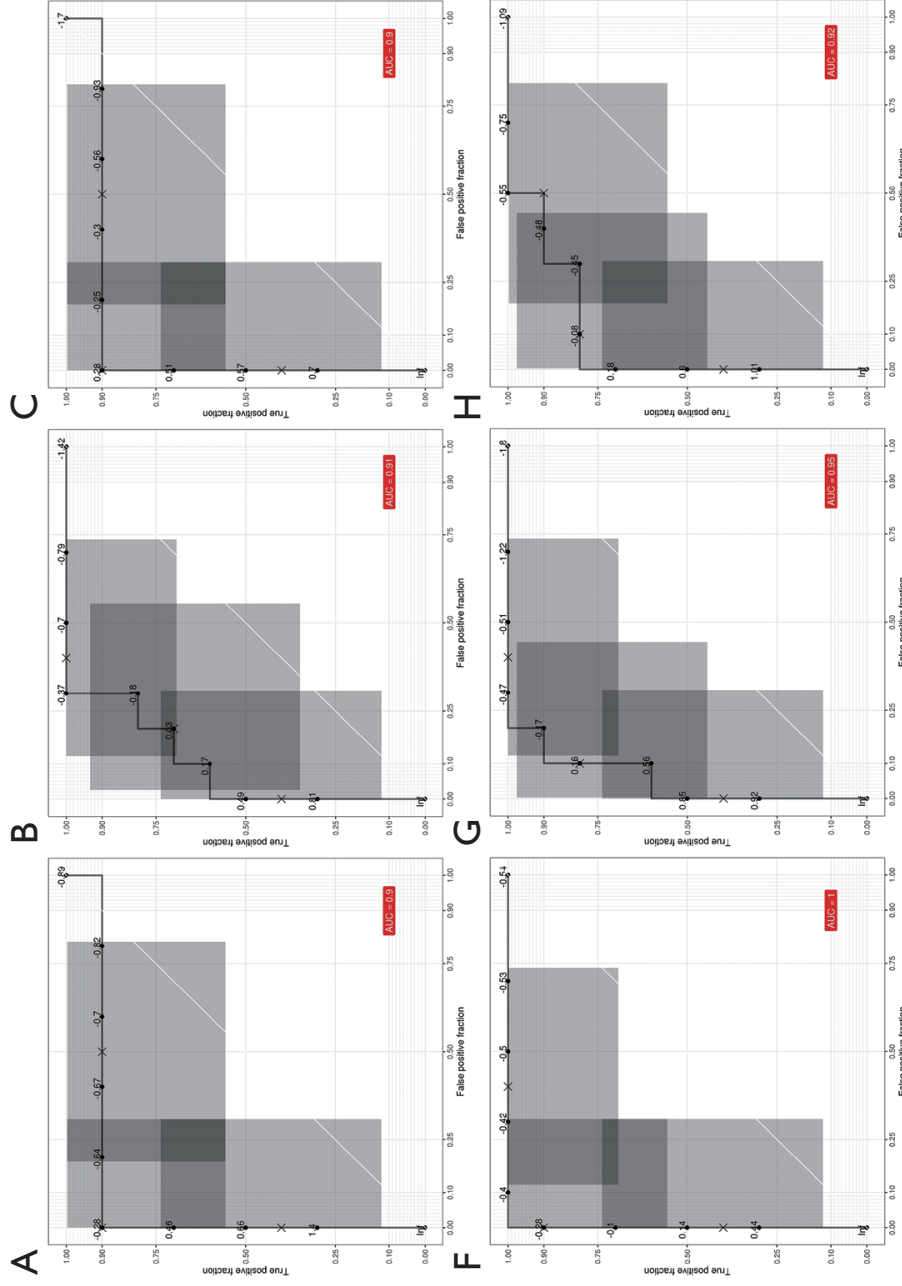
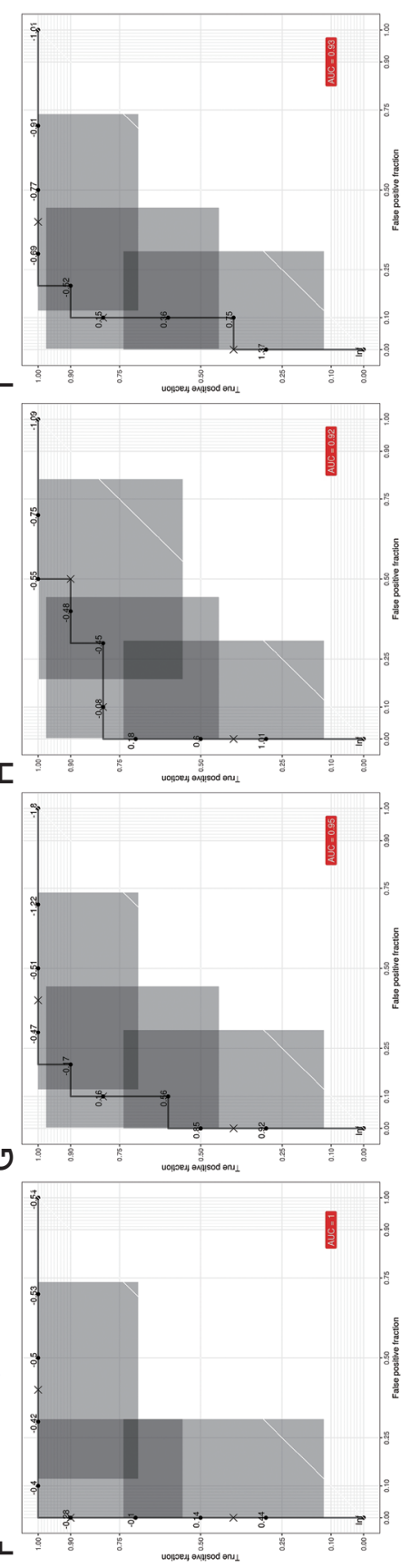
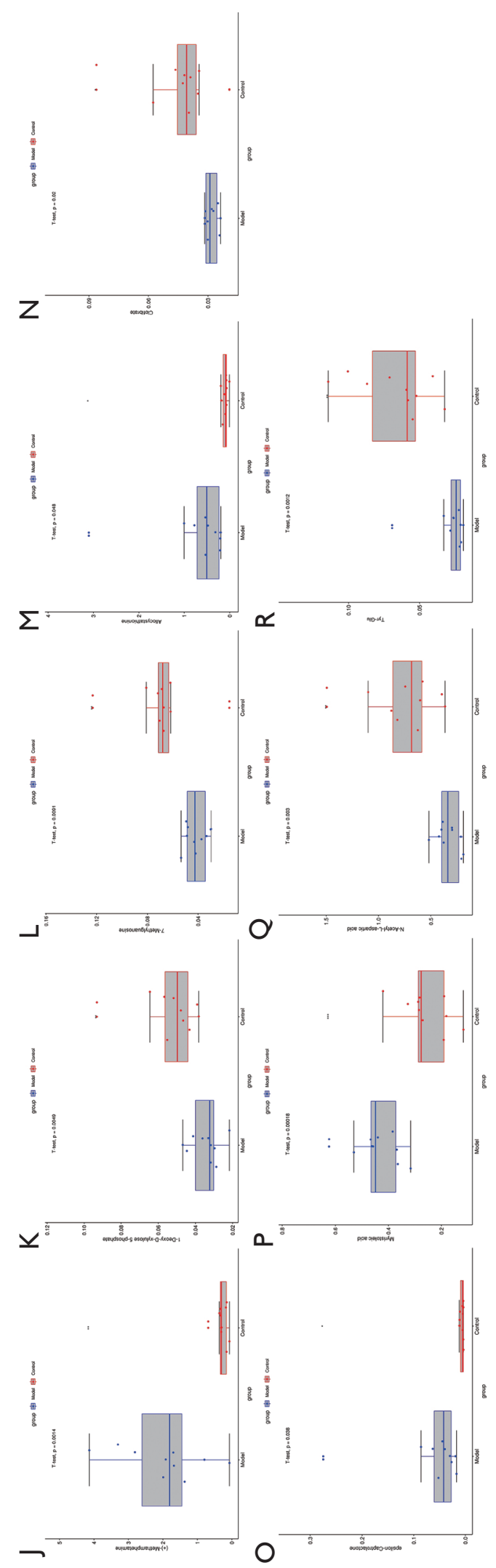

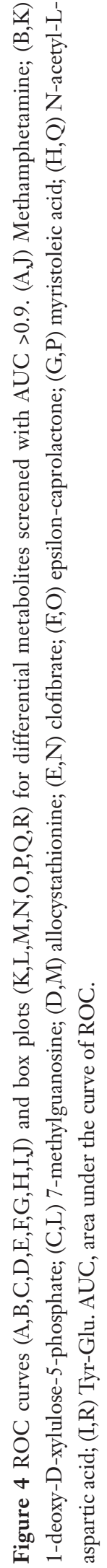



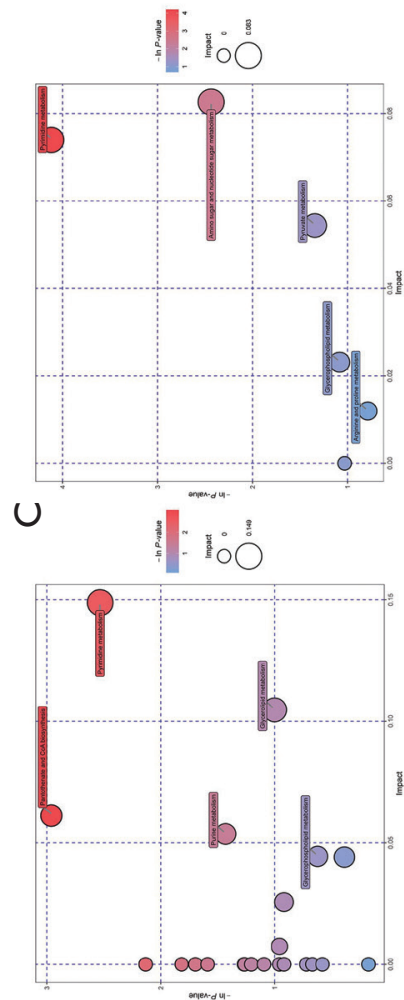

$\infty$

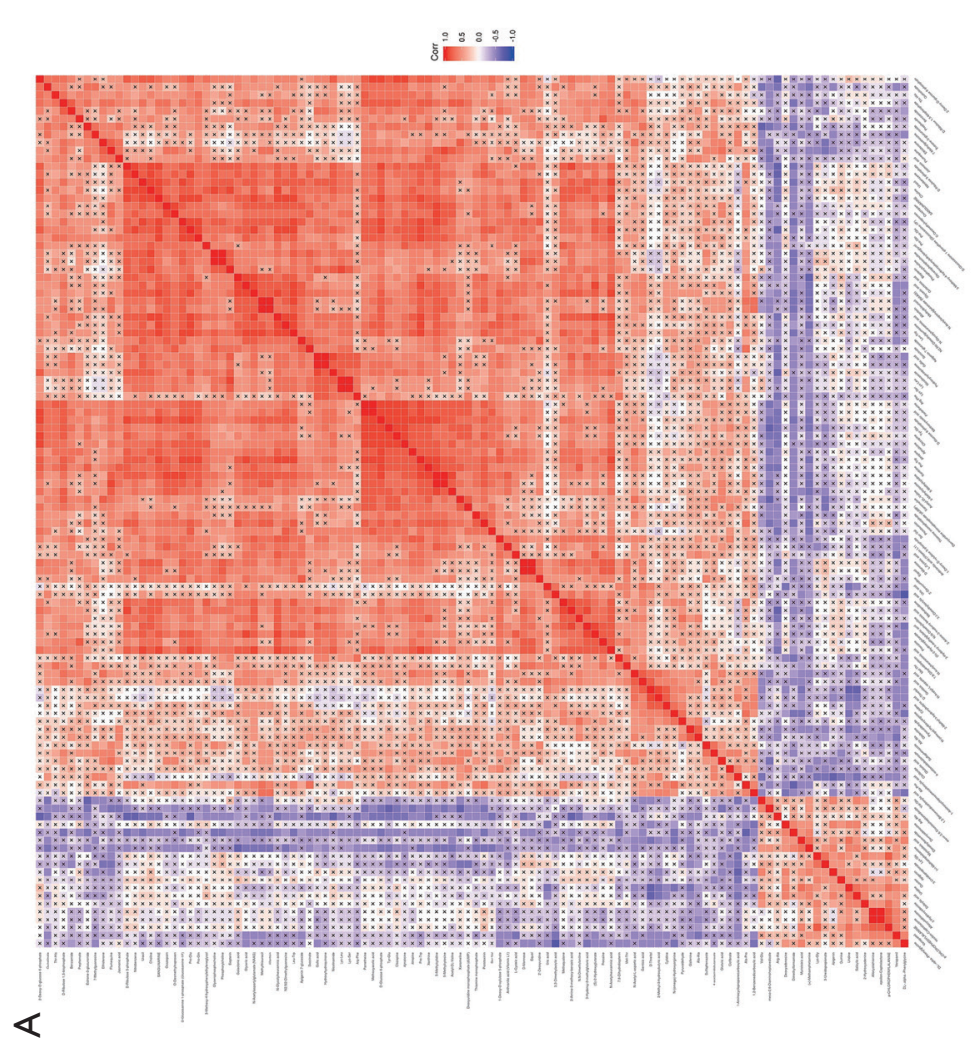

点

ш
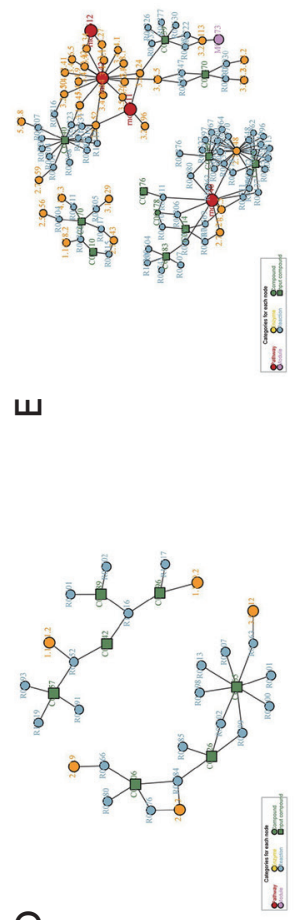

.

$\dot{s}$

을

to

चี

。

훙

음 되

$\frac{0}{0}$

है

के

菉

추

e

롱

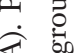

苟

苟

巳

조

긴

氙

t)

웅

党

苂

๐

吾

인

范

苂

苞

I

in.

离 
Table 2 Partial enriched pathway of differential metabolites

\begin{tabular}{llc}
\hline Pathway ID in KEGG database & Description of pathway & Compounds number \\
\hline rno01100 & Metabolic pathways & 46 \\
rno00240 & Pyrimidine metabolism & 8 \\
rno00520 & Amino sugar and nucleotide sugar metabolism & 6 \\
rno01230 & Biosynthesis of amino acids & 5 \\
rno00400 & Phenylalanine, tyrosine and tryptophan biosynthesis & 4 \\
\hline
\end{tabular}

and fibrosis. Further studies of metabolites, including targeted metabolomics studies, may generate meaningful information regarding signal pathways and pathogenesis.

Currently, ketamine-induced cystitis diagnosis mainly relies on a history of ketamine abuse and clinical symptoms as assessed by a urologist. Differential diagnosis can be hampered by patients not being upfront about their drug abuse history or if clinical symptoms are missed. To date, no urine biomarkers have been developed for the clinical detection of bladder dysfunction and fibrosis. Early diagnosis in patients with bladder fibrosis is one of the key factors in improving clinical outcomes. Some reports have already provided evidences about the application of urine metabolites in urinary diseases, such as interstitial cystitis, chronic prostatitis, bladder cancer and other urological disorders (26-28). In the ROC curves, metabolites with a large AUC values were identified as promising potential biomarkers for the ketamine-induced bladder rat model. These urinary metabolites may be potential biomarker in the clinical diagnosis of ketamine induced bladder dysfunction and the early detection of bladder fibrosis, and further research in animal models and ketamine abusers are warranted.

\section{Conclusions}

A rat model of ketamine-induced bladder fibrosis was successfully established. The expressions of urine metabolites in this model were analyzed through metabolomic method. A total of 118 differential metabolites were detected, 16 of which were up-regulated and 102 were down-regulated. Combined with bioinformatics approaches, the biological functions of the differential urine metabolites were analyzed. Related pathways included pyrimidine metabolism, amino sugar, phenylalanine, tyrosine and tryptophan biosynthesis, etc. It provided vital clues for understanding the pathogenesis, and further aids in the diagnosis and treatment of ketamine-induced bladder dysfunction and fibrosis.

\section{Acknowledgments}

The authors are grateful to Biotree Biotech (Co., Ltd., Shanghai, China) for technical support.

Funding: This work was supported by the National Natural Science Foundation of China (grant No. 81700667).

\section{Footnote}

Reporting Checklist: The authors have completed the ARRIVE reporting checklist. Available at http://dx.doi. org/10.21037/tau-20-1202

Data Sharing Statement: Available at http://dx.doi. org/10.21037/tau-20-1202

Conflicts of Interest: All authors have completed the ICMJE uniform disclosure form (available at http://dx.doi. org/10.21037/tau-20-1202). The authors have no conflicts of interest to declare.

Ethical Statement: The authors are accountable for all aspects of the work in ensuring that questions related to the accuracy or integrity of any part of the work are appropriately investigated and resolved. Experiments were performed under a project license (No. 2018sydw0225) granted by experimental animal welfare center in Central South University, Hunan, China, in compliance with China's "Animal management regulations" for the care and use of animals.

Open Access Statement: This is an Open Access article distributed in accordance with the Creative Commons Attribution-NonCommercial-NoDerivs 4.0 International License (CC BY-NC-ND 4.0), which permits the non- 
commercial replication and distribution of the article with the strict proviso that no changes or edits are made and the original work is properly cited (including links to both the formal publication through the relevant DOI and the license). See: https://creativecommons.org/licenses/by-nc-nd/4.0/.

\section{References}

1. Bokor G, Anderson P. Ketamine: an update on its abuse. J Pharm Pract 2014;27:582-6.

2. Trujillo KA, Smith ML, Sullivan B, et al. The neurobehavioral pharmacology of ketamine: implications for drug abuse, addiction, and psychiatric disorders. ILAR J 2011;52:366-78.

3. Shahani R, Streutker C, Dickson B, et al. Ketamineassociated ulcerative cystitis: a new clinical entity. Urology 2007;69:810-2.

4. Chung SD, Wang CC, Kuo HC. Augmentation enterocystoplasty is effective in relieving refractory ketamine-related bladder pain. Neurourol Urodyn 2014;33:1207-11.

5. Hopcroft SA, Cottrell AM, Mason K, et al. Ureteric intestinal metaplasia in association with chronic recreational ketamine abuse. J Clin Pathol 2011;64:551-2.

6. Peng TR, Lee MC, Wu TW, et al. Suspected ketamineassociated lower urinary tract symptoms. Urol J 2014;11:1508-10.

7. Castellani D, Pirola G, Gubbiotti M, et al. What urologists need to know about ketamine-induced uropathy: A systematic review. Neurourol Urodyn 2020;39:1049-62.

8. Ou YL, Liu CY, Cha TL, et al. Complete reversal of the clinical symptoms and image morphology of ketamine cystitis after intravesical hyaluronic acid instillation: A case report. Medicine (Baltimore) 2018;97:e11500.

9. Wang J, Chen Y, Gu D, et al. Ketamine-induced bladder fibrosis involves epithelial-to-mesenchymal transition mediated by transforming growth factor- $\beta 1$. Am J Physiol Renal Physiol 2017;313:F961-72. Erratum in: Am J Physiol Renal Physiol. 2018 Feb 1;314(2):F317. doi: 10.1152/ajprenal.zh2-8423-corr.2017.

10. Wu P, Wang Q, Huang Z, et al. Clinical staging of ketamine-associated urinary dysfunction: a strategy for assessment and treatment. World J Urol 2016;34:1329-36.

11. Jhang JF, Hsu YH, Kuo HC. Possible pathophysiology of ketamine-related cystitis and associated treatment strategies. Int J Urol 2015;22:816-25.

12. Hiller K, Hangebrauk J, Jäger C, et al. MetaboliteDetector: comprehensive analysis tool for targeted and nontargeted
GC/MS based metabolome analysis. Anal Chem 2009;81:3429-39.

13. Evans AM, DeHaven CD, Barrett T, et al. Integrated, nontargeted ultrahigh performance liquid chromatography/electrospray ionization tandem mass spectrometry platform for the identification and relative quantification of the small-molecule complement of biological systems. Anal Chem 2009;81:6656-67.

14. Fischer AH, Jacobson KA, Rose J, et al. Hematoxylin and eosin staining of tissue and cell sections. CSH Protoc 2008;2008:pdb.prot4986.

15. Ozawa A, Sakaue M. New decolorization method produces more information from tissue sections stained with hematoxylin and eosin stain and masson-trichrome stain. Ann Anat 2020;227:151431.

16. Iguchi K, Okumura N, Usui S, et al. Myristoleic acid, a cytotoxic component in the extract from Serenoa repens, induces apoptosis and necrosis in human prostatic LNCaP cells. Prostate 2001;47:59-65.

17. Li Y, Zhou H, Zhang Y, et al. N-3-(oxododecanoyl)-Lhomoserine lactone promotes the induction of regulatory T-cells by preventing human dendritic cell maturation. Exp Biol Med (Maywood) 2015;240:896-903.

18. Hu ZY, Chang J, Guo FF, et al. The effects of dimethylformamide exposure on liver and kidney function in the elderly population: A cross-sectional study. Medicine (Baltimore) 2020;99:e20749.

19. Moraes JP, Pereira DS, Matos AS, et al. The ethanol extract of the inner bark of Caesalpinia pyramidalis (Tul.) reduces urinary bladder damage during cyclophosphamideinduced cystitis in rats. ScientificWorldJournal 2013;2013:694010.

20. Bennett AH, Delrio A. Idiopathic rupture of the bladder: association with methamphetamine and alcohol. J Urol 1980;124:429-30.

21. Delgado JH, Caruso MJ, Waksman JC, et al. Acute, transient urinary retention from combined ecstasy and methamphetamine use. J Emerg Med 2004;26:173-5.

22. Cui L, Jiang X, Zhang C, et al. Ketamine induces endoplasmic reticulum stress in rats and SV-HUC-1 human uroepithelial cells by activating NLRP3/TXNIP aix. Biosci Rep 2019;39:BSR20190595.

23. Kim A, Yu H, Heo J, et al. Mesenchymal stem cells protect against the tissue fibrosis of ketamine-induced cystitis in rat bladder. Sci Rep 2016;6:30881.

24. Tanioka A, Deguchi T. Effect of a Bicyclic Pyrimidine Derivative (KRP-103), a Novel Selective Tachykinin NK1 Receptor Antagonist, on Bladder Function in Guinea Pigs. 
Drug Res (Stuttg) 2017;67:302-7.

25. Wada M, Wada Y, Uchiyama M, et al. (13)C-phenylalanine breath test correlates with liver fibrosis in postoperative biliary atresia. Pediatr Int 2007;49:836-41.

26. Kind T, Cho E, Park T, et al. Interstitial CystitisAssociated Urinary Metabolites Identified by MassSpectrometry Based Metabolomics Analysis. Sci Rep 2016;6:39227.

27. Shoskes DA, Altemus J, Polackwich AS, et al. The Urinary
Microbiome Differs Significantly Between Patients With Chronic Prostatitis/Chronic Pelvic Pain Syndrome and Controls as Well as Between Patients With Different Clinical Phenotypes. Urology 2016;92:26-32.

28. Witzke KE, Großerueschkamp F, Jütte H, et al. Integrated Fourier Transform Infrared Imaging and Proteomics for Identification of a Candidate Histochemical Biomarker in Bladder Cancer. Am J Pathol 2019;189:619-31.
Cite this article as: $\mathrm{Li} \mathrm{H}$, Zhu Q, Li K, Wu Z, Tang Z, Wang Z. Investigation of urinary components in rat model of ketamineinduced bladder fibrosis based on metabolomics. Transl Androl Urol 2021;10(2):830-840. doi: 10.21037/tau-20-1202 
Table S1 All differential metabolites in ketamine treated rat urine samples compared to control group

\begin{tabular}{|c|c|c|c|c|}
\hline MS2 name & Type & VIP & $P$ value & Fold change \\
\hline p-Chlorophenylalanine & MS2 reverse & 1.482664661 & 0.022581683 & 2.088403489 \\
\hline Myristoleic acid & MS2 reverse & 2.09799238 & 0.000179994 & 1.726790289 \\
\hline Salicylic acid & MS2 forward & 1.824331269 & 0.010147158 & 1.591570624 \\
\hline L-homoserine & MS2 reverse & 1.746636855 & 0.010277906 & 1.548048138 \\
\hline 3-Methoxy-4-hydroxyphenylethyleneglycol & MS2 reverse & 1.370359686 & 0.034436936 & 0.829686969 \\
\hline Sulfaphenazole & MS2 reverse & 1.029843202 & 0.03273948 & 0.795389004 \\
\hline 1,2-Benzenedicarboxylic acid & MS2 forward & 1.5430191 & 0.045110528 & 0.777020585 \\
\hline Quinolinate & MS2 forward & 2.082448204 & 0.00236724 & 0.775982736 \\
\hline D-glucosamine 1-phosphate & MS2 forward & 1.833854214 & 0.008842721 & 0.771064659 \\
\hline Phosphorylcholine & MS2 forward & 1.117232921 & 0.028263596 & 0.769568172 \\
\hline D-ribulose 5-phosphate & MS2 forward & 1.780321548 & 0.018475696 & 0.768976317 \\
\hline Allantoin & MS2 forward & 2.046300074 & 0.010595984 & 0.762709318 \\
\hline Glyceric acid & MS2 reverse & 2.121688111 & 0.01057002 & 0.761944433 \\
\hline Galactonic acid & MS2 forward & 1.951851055 & 0.0313966 & 0.758280251 \\
\hline Picrotoxinin & MS2 reverse & 2.079133994 & 0.018233279 & 0.755109009 \\
\hline Thiamine monophosphate & MS2 reverse & 2.063228119 & 0.006774628 & 0.75347008 \\
\hline N-Acetylaspartylglutamate (NAAG) & MS2 reverse & 2.291827297 & 0.003445462 & 0.749532403 \\
\hline Methylthiouracil & MS2 reverse & 2.160625989 & 0.005118714 & 0.746049357 \\
\hline Glutaric acid & MS2 reverse & 1.557278238 & 0.047708268 & 0.745016803 \\
\hline L-Cysteic acid & MS2 reverse & 1.623170822 & 0.04716071 & 0.741823206 \\
\hline 3',5'-Cyclic guanosine monophosphate & MS2 forward & 1.710857486 & 0.020098057 & 0.730690388 \\
\hline N-Acetylneuraminic acid & MS2 forward & 1.749960452 & 0.033525987 & 0.728765601 \\
\hline Biopterin & MS2 forward & 1.227077427 & 0.044781799 & 0.722976261 \\
\hline Hydroxyproline & MS2 reverse & 1.901941039 & 0.031785026 & 0.719187455 \\
\hline (S)-2-Hydroxyglutarate & MS2 forward & 1.610349719 & 0.034992987 & 0.710700459 \\
\hline D-Glucose 6-phosphate & MS2 reverse & 2.086381705 & 0.004901267 & 0.685505863 \\
\hline 3-Hydorxy-3-methylglutaric acid & MS2 forward & 1.501667044 & 0.046499813 & 0.67649715 \\
\hline Xanthine & MS2 forward & 2.091674633 & 0.011043818 & 0.674563903 \\
\hline Gallic acid & MS2 forward & 1.468370852 & 0.01298651 & 0.669149235 \\
\hline Succinate & MS2 forward & 1.649946217 & 0.021349092 & 0.665581289 \\
\hline Uracil & MS2 forward & 1.52020403 & 0.022438883 & 0.659230513 \\
\hline 3-Hydroxyisovaleric acid & MS2 reverse & 1.667482832 & 0.021694764 & 0.647310844 \\
\hline Allocystathionine & MS2 reverse & 1.883617934 & 0.038591377 & 0.646847067 \\
\hline 2'-Deoxyuridine & MS2 reverse & 1.230308737 & 0.040283808 & 0.620371895 \\
\hline Hydroxyhydroquinone & MS2 reverse & 1.662096942 & 0.007088873 & 0.618218772 \\
\hline Deoxyuridine monophosphate & MS2 reverse & 1.649252621 & 0.025565738 & 0.614569781 \\
\hline Jasmonic acid & MS2 reverse & 1.514285312 & 0.036044238 & 0.610840674 \\
\hline Pantetheine & MS2 forward & 1.675574703 & 0.022016291 & 0.600185386 \\
\hline Apigenin 7-glucoside & MS2 reverse & 1.580735469 & 0.021473258 & 0.574226734 \\
\hline Prephenate & MS2 reverse & 1.358369196 & 0.014242113 & 0.567506361 \\
\hline 2-Amino-3-methoxy-benzoic acid & MS2 reverse & 1.787397399 & 0.017242521 & 0.561417149 \\
\hline 5-Hydroxymethylcytidine & MS2 reverse & 1.396897031 & 0.019620982 & 0.556911219 \\
\hline L-Ascorbic acid & MS2 forward & 1.413277202 & 0.048514968 & 0.5558384 \\
\hline D-Ribulose 1,5-bisphosphate & MS2 reverse & 2.031981268 & 0.015979171 & 0.550204342 \\
\hline Oxazepam & MS2 reverse & 1.860735515 & 0.039675759 & 0.54668473 \\
\hline L-Histidinol & MS2 reverse & 1.613477995 & 0.021146814 & 0.53101848 \\
\hline D-Threitol & MS2 forward & 1.902102624 & 0.016490356 & 0.530728427 \\
\hline Uric acid & MS2 forward & 1.720076229 & 0.045187868 & 0.517918642 \\
\hline Glafenine & MS2 reverse & 2.137081115 & 0.005373777 & 0.5110753 \\
\hline Clozapine & MS2 reverse & 1.939712787 & 0.025412069 & 0.499340036 \\
\hline 2-Deoxy-D-glucose 6-phosphate & MS2 reverse & 1.656468689 & 0.039200661 & 0.495137978 \\
\hline Estrone-3-glucuronide & MS2 reverse & 2.097005256 & 0.006118495 & 0.487421812 \\
\hline 2-Methyl-3-hydroxybutyric acid & MS2 reverse & 1.72070876 & 0.037063118 & 0.486247008 \\
\hline N-Acetyl-L-aspartic acid & MS2 reverse & 2.398347827 & 0.002969733 & 0.436451439 \\
\hline Anthranilic acid (Vitamin L1) & MS2 reverse & 1.858261397 & 0.030461366 & 0.355221406 \\
\hline Hydroxyphenyllactic acid & MS2 reverse & 2.633650228 & 0.019664464 & 17.03006355 \\
\hline Epsilon-caprolactone & MS2 reverse & 2.477916359 & 0.038380883 & 9.592274572 \\
\hline Allocystathionine & MS2 reverse & 2.172458016 & 0.0480407 & 7.017815453 \\
\hline
\end{tabular}

Table S1 (continued) 
Table S1 (continued)

\begin{tabular}{|c|c|c|c|c|}
\hline MS2 name & Type & VIP & $P$ value & Fold change \\
\hline (+)-Methamphetamine & MS2 forward & 2.347313599 & 0.001415391 & 6.949027624 \\
\hline Dimethylformamide & MS2 reverse & 1.940048907 & 0.006059808 & 4.206489916 \\
\hline Meso-2,6-diaminoheptanedioate & MS2 reverse & 1.257826352 & 0.023069712 & 2.675236496 \\
\hline Uridine & MS2 reverse & 2.020893568 & 0.003712476 & 1.699312722 \\
\hline Verapamil & MS2 reverse & 2.16242187 & 0.002288484 & 1.683733074 \\
\hline Lys-Gly & MS2 reverse & 1.473315216 & 0.0448077 & 1.520242251 \\
\hline Arg-Ala & MS2 reverse & 1.377219343 & 0.0099348 & 1.301056568 \\
\hline Val-Glu & MS2 reverse & 1.718494154 & 0.025726621 & 1.2977372 \\
\hline DL-alpha-phenylglycine & MS2 reverse & 1.694713395 & 0.014759062 & 1.183587801 \\
\hline 4-acetamidobutanoate & MS2 forward & 1.624612075 & 0.008246869 & 0.843757376 \\
\hline O-Desmethylnaproxen & MS2 reverse & 1.520776335 & 0.005264959 & 0.769378923 \\
\hline 5-Methylcytosine & MS2 forward & 1.64652063 & 0.013511683 & 0.76512237 \\
\hline Thymidine & MS2 reverse & 1.496465311 & 0.017604915 & 0.761783666 \\
\hline Acetylglycine & MS2 reverse & 1.255082407 & 0.020875357 & 0.759055512 \\
\hline Pro-Glu & MS2 forward & 1.476630987 & 0.033605868 & 0.75816417 \\
\hline Thr-His & MS2 reverse & 1.220499196 & 0.036751716 & 0.751130341 \\
\hline 5-Methylcytidine & MS2 forward & 1.658484074 & 0.014583692 & 0.745343053 \\
\hline Nitrobenzene & MS2 reverse & 1.209341788 & 0.03705139 & 0.744022402 \\
\hline 3,3-Dimethylacrylic acid & MS2 forward & 1.589224968 & 0.02691495 & 0.740013662 \\
\hline Ser-Val & MS2 reverse & 1.555183809 & 0.024983138 & 0.738083133 \\
\hline $\mathrm{N}$-(omega)-Hydroxyarginine & MS2 reverse & 1.333257353 & 0.009896441 & 0.730639649 \\
\hline Glycerophosphocholine & MS2 forward & 1.078812605 & 0.018463782 & 0.729971001 \\
\hline Sanguinarine & MS2 reverse & 1.494057237 & 0.043342222 & 0.723800293 \\
\hline Anthranilic acid (vitamin L1) & MS2 reverse & 1.163473953 & 0.02848806 & 0.717913533 \\
\hline Atropine & MS2 reverse & 1.679877549 & 0.004653833 & 0.712118058 \\
\hline N2,N2-Dimethylguanosine & MS2 reverse & 1.755659554 & 0.01585386 & 0.7087619 \\
\hline N-Acetyl-D-glucosamine & MS2 forward & 1.629226838 & 0.01782848 & 0.707239534 \\
\hline Biopterin & MS2 forward & 1.000834999 & 0.042091843 & 0.705518001 \\
\hline Pro-Trp & MS2 reverse & 1.793857835 & 0.014064219 & 0.704400066 \\
\hline Cytidine & MS2 reverse & 1.574311055 & 0.018826082 & 0.703354023 \\
\hline Flumequine & MS2 reverse & 1.813932221 & 0.010658893 & 0.703252572 \\
\hline Pyruvaldehyde & MS2 reverse & 1.142383824 & 0.049498601 & 0.700527032 \\
\hline Met-Thr & MS2 reverse & 1.249302443 & 0.021575958 & 0.694715977 \\
\hline Leu-Trp & MS2 reverse & 1.745869501 & 0.00711319 & 0.682205215 \\
\hline Xanomeline & MS2 reverse & 1.923823003 & 0.008476593 & 0.674795605 \\
\hline Ala-Pro & MS2 reverse & 1.579299618 & 0.005411376 & 0.661127047 \\
\hline N-GlycolyIneuraminic acid & MS2 reverse & 1.57156514 & 0.030426096 & 0.659859161 \\
\hline Acetyl-DL-Valine & MS2 reverse & 1.734203519 & 0.011445194 & 0.655074708 \\
\hline Ethisterone & MS2 reverse & 1.755203974 & 0.032942997 & 0.649340674 \\
\hline 1-Deoxy-D-xylulose 5-phosphate & MS2 reverse & 1.893722005 & 0.004923546 & 0.643672651 \\
\hline Clofibrate & MS2 reverse & 1.778152384 & 0.019533896 & 0.639236238 \\
\hline N-Acetylneuraminic acid & MS2 forward & 1.893214103 & 0.011852953 & 0.637754926 \\
\hline Procaine & MS2 reverse & 1.267212456 & 0.021033961 & 0.634484755 \\
\hline Methoxyacetic acid & MS2 reverse & 1.613501592 & 0.017177443 & 0.62354792 \\
\hline $\mathrm{N}, \mathrm{N}$-Dimethylaniline & MS2 reverse & 1.38670469 & 0.047154221 & 0.622392371 \\
\hline Lys-Leu & MS2 reverse & 1.226355959 & 0.026133676 & 0.621554911 \\
\hline Lys-Ser & MS2 reverse & 1.248923791 & 0.027392882 & 0.619395745 \\
\hline Ala-Ala & MS2 reverse & 1.563839603 & 0.00849707 & 0.606806261 \\
\hline 7-Methylguanosine & MS2 reverse & 1.712194102 & 0.009144798 & 0.603191597 \\
\hline Equol & MS2 forward & 1.255913117 & 0.030707226 & 0.588731875 \\
\hline Malonic acid & MS2 reverse & 1.965097321 & 0.004436536 & 0.583918122 \\
\hline Ajmalicine & MS2 reverse & 1.687588952 & 0.007489708 & 0.582624244 \\
\hline D-Mannose & MS2 forward & 1.286248655 & 0.033116293 & 0.563901819 \\
\hline Quinate & MS2 reverse & 1.422653095 & 0.039765564 & 0.559847402 \\
\hline Arg-Phe & MS2 forward & 1.665346043 & 0.010164035 & 0.493562524 \\
\hline 1-Aminocyclopropanecarboxylic acid & MS2 reverse & 1.78917087 & 0.017018907 & 0.458740484 \\
\hline 7,8-Dihydrobiopterin & MS2 forward & 1.424846621 & 0.010708745 & 0.430668925 \\
\hline Tyr-Glu & MS2 reverse & 2.119367582 & 0.00081498 & 0.428884596 \\
\hline
\end{tabular}

